\title{
An Investigation on the Beta Function III: Explicit Evaluation of the Harmonic Number $\mathrm{H}_{\frac{3}{2}}$
}

\section{Edigles Guedes ${ }^{1}$ and Prof. Dr. K. Raja Rama Gandhi ${ }^{2}$}

Number Theorist, Brazil ${ }^{1}$

Resource perosn in Mathematics for Oxford University Press and Professor at BITS-Vizag ${ }^{2}$

Abstract. In previous paper, I developed new versions of the Euler beta function, which given a closed form for the harmonic number $H_{\frac{3}{2}}$.

\section{INTRODUCTION}

In this paper, I prove that

$$
\sum_{k=1}^{\infty} \frac{(2 k) !}{4^{k} k !} \sum_{n=1}^{j} \frac{\Gamma\left(n+\frac{1}{2}\right)}{\Gamma\left(n+k+\frac{3}{2}\right)}=H_{j}-H_{j+\frac{1}{2}}+2-2 \ln 2,
$$

which provided the special value

$$
H_{\frac{3}{2}}=\frac{8}{3}-2 \ln 2
$$

\section{THEOREM}

THEOREM 1. I have

$$
H_{\frac{3}{2}}=\frac{8}{3}-2 \ln 2
$$

where $H_{j}$ is the harmonic number.

Proof. In [1], I prove that

$$
\frac{1}{n \Gamma\left(n+\frac{1}{2}\right)}=\sum_{k=0}^{\infty} \frac{(2 k) !}{4^{k} k ! \Gamma\left(n+k+\frac{3}{2}\right)} \Rightarrow \frac{1}{n}=\sum_{k=0}^{\infty} \frac{(2 k) ! \Gamma\left(n+\frac{1}{2}\right)}{4^{k} k ! \Gamma\left(n+k+\frac{3}{2}\right)}=\frac{2}{2 n+1}+\sum_{k=1}^{\infty} \frac{(2 k) ! \Gamma\left(n+\frac{1}{2}\right)}{4^{k} k ! \Gamma\left(n+k+\frac{3}{2}\right)}
$$

I sum in both sides of equation above from 1 at $j$ with respect to $n$

$$
\sum_{n=1}^{j} \frac{1}{n}=2 \sum_{n=1}^{j} \frac{1}{2 n+1}+\sum_{k=1}^{\infty} \frac{(2 k) !}{4^{k} k !} \sum_{n=1}^{j} \frac{\Gamma\left(n+\frac{1}{2}\right)}{\Gamma\left(n+k+\frac{3}{2}\right)}
$$

ergo,

$$
\sum_{k=1}^{\infty} \frac{(2 k) !}{4^{k} k !} \sum_{n=1}^{j} \frac{\Gamma\left(n+\frac{1}{2}\right)}{\Gamma\left(n+k+\frac{3}{2}\right)}=H_{j}-H_{j+\frac{1}{2}}+2-2 \ln 2
$$

Let $j=1$ in Theorem 1 , then

$$
H_{\frac{3}{2}}=\frac{8}{3}-2 \ln 2
$$


I not can calculate other special values, since, for $j>1$, the summation

$$
\sum_{k=1}^{\infty} \frac{(2 k) !}{4^{k} k !} \sum_{n=1}^{j} \frac{\Gamma\left(n+\frac{1}{2}\right)}{\Gamma\left(n+k+\frac{3}{2}\right)}
$$

is a divergent series. $\square$

\section{REFERENCES}

[1] Guedes, Edigles, An Investigation on the Beta Function II: The Summations of 1/ $\sqrt{\pi}, 2013$. 